\title{
AVALIAÇÃO DA INFLUÊNCIA DE SULFETOS SOLÚVEIS NA COMPLEXAÇÃO DO COBRE EM ÁGUAS SUPERFICIAIS EMPREGANDO MÉTODOS VOLTAMÉTRICOS
}

\author{
Elizabeth W. O. Scheffer \\ Departamento de Química, Universidade Estadual de Ponta Grossa, Av. Carlos Cavalcanti, 4748, 84030-000 Ponta Grossa - PR, Brasil \\ Marco T. Grassi* \\ Departamento de Química, Universidade Federal do Paraná, CP 19081, 81531-990 Curitiba - PR, Brasil
}

Recebido em 18/5/09; aceito em 5/2/10; publicado na web em 21/5/10

\begin{abstract}
INFLUENCE OF DISSOLVED SULFIDES ON COPPER COMPLEXATION IN SURFACE WATERS USING VOLTAMMETRIC METHODS. In this work a sulfide quantification protocol using voltammetric methods was developed to evaluate the effect of dissolved sulfides on copper complexation. On the basis of $\mathrm{pH}$, sulfide release from the dissociation of specific metal sulfide complexes can be electrochemically measured and then removed $\left(\mathrm{as}_{2} \mathrm{~S}\right.$ ) by a $\mathrm{N}_{2}$ purge. Cathodic stripping square wave voltammetry (CSSWV) was conducted to quantify $\mathrm{Cu}$ sulfides complexes which dissociate at $\mathrm{pH}<5.0$ during the process of acid titration.
\end{abstract}

Keywords: sulfide; copper speciation; square wave voltammetry.

\section{INTRODUÇÃO}

A presença de sulfetos em águas naturais e a sua importância nesse meio como ligante para metais, frequentemente relatada para ambientes anóxicos e associada à formação de compostos insolúveis, tem merecido destaque na literatura também pela sua relevância em águas superficiais ricas em oxigênio. ${ }^{1-5}$ Nestes ambientes, a formação de complexos solúveis de sulfeto metálico mostra-se responsável pela estabilização do sulfeto de hidrogênio em solução e, principalmente, desempenha um potencial controle sobre a disponibilidade de metais traço. ${ }^{6}$

Entretanto, apesar de seu importante significado biogeoquímico, existem relativamente poucos dados para níveis traço $(<1 \mu \mathrm{M})$ relacionados com a presença de sulfeto dissolvido em ambientes aquáticos, onde as concentrações estão na faixa de picomol a milimol por litro. ${ }^{7}$

De acordo com Bowles e colaboradores, ${ }^{3}$ a maioria dos dados conhecidos para sulfetos foi obtida usando o método colorimétrico convencional que tem sensibilidade de $\mathrm{mmol} \mathrm{L}^{-1}$, enquanto que as técnicas voltamétricas são confiáveis quantitativamente, em níveis de nmol L-1.

Os métodos voltamétricos são considerados adequadamente sensíveis e seletivos para determinar a concentração de íons metálicos livres nas baixas concentrações encontradas, por exemplo, em águas naturais. Entre as suas características merecem destaque além do excelente limite de detecção em concentrações de até nmol L ${ }^{-1}$, uma ótima correlação com a disponibilidade biológica de espécies metálicas., ${ }^{4,8}$

A voltametria, em combinação com diferentes técnicas espectroscópicas e cromatográficas, tem sido empregada para gerar não apenas informações sobre concentrações, mas também sobre a identidade e o comportamento das espécies de enxofre como, por exemplo, a formação de metalocomplexos em águas naturais. ${ }^{9}$

A voltametria de onda quadrada (VOQ) está entre os métodos que mais têm se mostrado apropriados para a determinação de sulfetos em uma ampla variedade de amostras, tais como águas naturais, alimentos e diferentes produtos da indústria do petróleo. ${ }^{9} \mathrm{O}$ voltamograma obtido mostra excelente sensibilidade e uma significativa redução nas correntes residuais.

Todas estas características já consagradas dos métodos voltamétricos foram fundamentais para o desenvolvimento de um protocolo inédito para detecção de níveis traços de metal e de sulfeto por Rozan

\footnotetext{
*e-mail: mtgrassi@quimica.ufpr.br
}

e colaboradores. ${ }^{10}$ Empregando voltametria de redissolução catódica com onda quadrada (VRCOQ) durante procedimento de titulação ácida das amostras, demonstraram a complexação do sulfeto com metais tais como $\mathrm{Mn}, \mathrm{Fe}, \mathrm{Co}, \mathrm{Ni}, \mathrm{Cu}$ e $\mathrm{Zn}$, em níveis de nanomol.

Visto o interesse em avaliar a complexação do cobre pelo sulfeto em amostras naturais, buscou-se aplicar o protocolo desenvolvido por Rozan e colaboradores. ${ }^{10}$ Entretanto, isto só foi possível a partir de sua adaptação, pois embora tenha mostrado sensibilidade e permitido a identificação dos diferentes metais complexados ao sulfeto, constatouse que alguns fatores não considerados no referido protocolo poderiam tornar o sinal analítico não indicativo das reais concentrações de metal complexado ao sulfeto: alterações no meio, determinadas pela mudança do pH durante a titulação ácida e, a complexidade da matriz em estudo.

Os estudos realizados neste trabalho para avaliar possíveis fatores interferentes permitiram importantes avanços com relação ao protocolo inicial. A construção de curvas analíticas foi uma modificação imprescindível para que se pudesse alcançar o principal objetivo de implantar um protocolo para avaliar a influência do sulfeto em concentrações de nmol L $\mathrm{L}^{-1}$ através da voltametria. Nesta nova abordagem considerou-se a relevância da suscetibilidade deste método às variações nas condições analíticas e a complexidade das amostras estudadas.

\section{PARTE EXPERIMENTAL}

Amostras de águas naturais foram coletadas em locais escolhidos por serem representativos de diversos níveis de impactação da expansão urbana sobre a Bacia do Alto Iguaçu. Os processos de coleta, preservação e estocagem foram desenvolvidos conforme descrito por Scheffer e colaboradores. ${ }^{1}$

A identificação de $\mathrm{CuS}$ nas amostras foi realizada por VRCOQ em alíquotas filtradas. Os parâmetros instrumentais empregados foram: eletrodeposição a $-0,1 \mathrm{~V}$ durante $180 \mathrm{~s}$ e varredura de potencial de $-0,1$ até $-1,4 \mathrm{~V}$ a $200 \mathrm{mV} \mathrm{s}^{-1}$, com frequência de $100 \mathrm{~Hz}$ e amplitude de pulso de $50 \mathrm{mV}$ sob força iônica do meio ajustada em $0,1 \mathrm{~mol} \mathrm{~L}^{-1}$ com $\mathrm{KNO}_{3}$ (Merck). ${ }^{1}$ A determinação da concentração de sulfetos foi feita em um potenciostato EG\&G PAR M394 acoplado a um sistema de eletrodos SMDE EG\&G PAR 303A utilizando o modo gota pendente de mercúrio. Este eletrodo operou com tamanho médio de gota e no modo HMDE. 
O procedimento empregado para a identificação de $\mathrm{CuS}$ através de titulação ácida inclui uma série de adições de ácido e purgas com $\mathrm{N}_{2}$ permitindo realizar a determinação semiquantitativa do cobre ligado ao sulfeto nas amostras de águas naturais, através do monitoramento do pico de redissolução do sulfeto em pH 5,0 e 2,8 realizado imediatamente após a acidificação. Neste intervalo de pH ocorre a dissociação dos complexos de sulfeto de cobre.

A vazão do nitrogênio durante as purgas foi em torno de $70 \mathrm{~mL}$ $\min ^{-1}$. Os volumes de $\mathrm{HNO}_{3}$ necessários para o ajuste do $\mathrm{pH}$ durante a titulação voltamétrica dependeram, entre outros fatores, do volume da amostra a ser acidificado, e das características fisico-químicas de cada amostra, tais como alcalinidade, $\mathrm{pH}$ natural e conteúdo de COD. ${ }^{1}$

Cabe salientar que todo o desenvolvimento do trabalho se deu sob um estrito regime de controle e garantia de qualidade, com avaliação periódica de controles (brancos) e reagentes, de maneira a minimizar a ocorrência de contaminações ou perda dos analitos.

\section{RESULTADOS E DISCUSSÃO}

\section{Implantação do método para avaliar a contribuição dos sulfetos solúveis na complexação do cobre}

A determinação das concentrações de complexos de sulfeto de cobre empregando técnicas voltamétricas foi realizada durante procedimento de titulação ácida, na qual a dissociação de sulfetos e metais ocorre em faixas específicas de $\mathrm{pH}$. Rozan e colaboradores ${ }^{10}$ desenvolveram um protocolo com base no $\mathrm{pH}$ de dissociação de complexos metálicos com sulfetos, sugerindo que estes podem ser agrupados em três zonas distintas (Tabela 1).

Tabela 1. Relação dos principais sulfetos metálicos que sofrem dissociação durante o procedimento de titulação ácida das amostras de águas naturais. Adaptada da ref. 10

\begin{tabular}{|c|c|c|c|}
\hline Grupos & $\mathrm{pH}$ & $\begin{array}{c}\text { Espécies } \\
\text { dissociadas }\end{array}$ & Kps \\
\hline I & $>6,7$ & $\begin{array}{c}\text { Sulfetos de } \\
\mathrm{Mn}^{2+}, \mathrm{Fe}^{2+}, \mathrm{Co}^{2+} \\
\text { e } \mathrm{Ni}^{2+}\end{array}$ & $\begin{aligned} \mathrm{MnS} & =3 \cdot 10^{-14} \\
\mathrm{FeS} & =8 \cdot 10^{-19} \\
\mathrm{CoS} & =5 \cdot 10^{-22} \\
\mathrm{NiS} & =4 \cdot 10^{-20}\end{aligned}$ \\
\hline II & $5,0-6,7$ & $\begin{array}{c}\text { Sulfetos de } \\
\mathrm{Zn}^{2+}, \mathrm{Pb}^{2+} \mathrm{e} \mathrm{Cd}^{2+}\end{array}$ & $\begin{array}{l}\mathrm{ZnS}=2 \cdot 10^{-25} \\
\mathrm{PbS}=3 \cdot 10^{-28} \\
\mathrm{CdS}=1 \cdot 10^{-27}\end{array}$ \\
\hline III & $<5,0$ & Sulfetos de $\mathrm{Cu}^{2+}$ & $\mathrm{CuS}=8 \cdot 10^{-37}$ \\
\hline
\end{tabular}

A determinação do sulfeto por VRCOQ originou voltamogramas onde podem ser observados os picos relativos às espécies eletroativas de sulfeto na amostra, em diferentes valores de $\mathrm{pH}$, durante o procedimento de titulação ácida. O pico inicial, observado em aproximadamente $-0,6 \mathrm{~V}$ antes da acidificação, representou todas as espécies eletroativas de enxofre, tais como $\mathrm{S}^{0}, \mathrm{HS}, \mathrm{S}^{2-}, \mathrm{S}_{\mathrm{x}}{ }^{2-}$, que podem estar presentes na amostra em $\mathrm{pH}$ maior do que 6,7.

Após acidificação, em cada zona de pH (Tabela 1) sulfetos metálicos que sofrem dissociação foram medidos eletroquimicamente e, então, removidos na forma de $\mathrm{H}_{2} \mathrm{~S}$ pela purga com $\mathrm{N}_{2}$. Visto que o $\mathrm{pH}$ inicial das amostras neste trabalho situou-se entre 5,7 e 7,6 certos complexos podem, em alguns casos, não estar presentes uma vez que o pH não se mostrou favorável.

Após a medida do pico de sulfeto em $\mathrm{pH}$ 5,0 purgou-se a solução por 300 s e procedeu-se nova leitura, observando-se um pico de menor intensidade que corresponde apenas a $\mathrm{S}^{0}$, restante da etapa anterior ou da dissociação de polissulfetos, que ocorre em $\mathrm{pH}<6,5$ para $\mathrm{S}_{4}{ }^{2-}$ e $\mathrm{pH}<6,1$ para $\mathrm{S}_{5}^{2-}$. Seguindo-se o protocolo de titulação ácida, a amostra foi em seguida acidificada para $\mathrm{pH}$ 2,8 sem purga, quando o sulfeto de $\mathrm{Cu}$ presente na amostra sofre dissociação e o sulfeto liberado é medido juntamente com o $\mathrm{S}^{0}$ restante da etapa anterior. ${ }^{10}$ $\mathrm{O}$ aumento na intensidade do pico em aproximadamente $-0,6 \mathrm{~V}$, e a diferença dos valores de corrente, entre $\mathrm{pH}$ 5,0 e 2,8 permitiu indicar $\mathrm{S}^{2-}$ ligado ao cobre.

\section{Determinação em paralelo da concentração de cobre dissociado}

Na etapa inicial da implantação da metodologia neste trabalho, buscou-se, paralelamente, durante a titulação ácida para sulfeto a determinação do cobre dissolvido através de VRAPD. Neste procedimento, após a última acidificação da amostra a pH 2,8 iniciava-se a adição do padrão de cobre e, dessa maneira, determinava-se a concentração do metal complexado pelo sulfeto. Uma vez conhecida a concentração de cobre em pH 2,8 calculava-se a concentração em pH 5,0 considerando-se os valores de corrente obtidos. A diferença entre as duas concentrações indicava a concentração de cobre ligado ao sulfeto que se dissociou pela acidificação da amostra.

De maneira que o aumento da corrente para o cobre após a acidificação em $\mathrm{pH}$ 2,8 foi inicialmente atribuído ao aumento da concentração deste íon pela dissociação dos complexos de sulfeto de cobre, que ocorre em valores de $\mathrm{pH}$ inferiores a 5,0 (Figura 1).

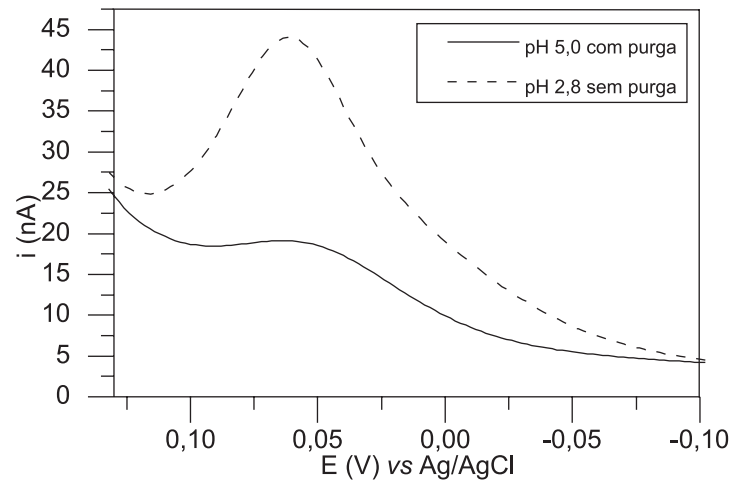

Figura 1. Voltamogramas para determinação de cobre por VRAPD durante titulação ácida. Parâmetros de análise: ajuste força iônica $\mathrm{KNO}_{3} 0,1 \mathrm{~mol} \mathrm{~L}^{-1}$; $E_{i}=-0,6 \mathrm{~V}(\mathrm{vs} \mathrm{Ag} / \mathrm{AgCl}) ; E_{f}=0,15 \mathrm{~V} ; E_{\text {cond }}=-1,2 \mathrm{~V} ; t_{\text {dep }}=600 \mathrm{~s}$; amplitude do pulso $=50 \mathrm{mV}$; velocidade de varredura $=4 \mathrm{mV} \mathrm{s}^{-1}$

Esta relação entre a corrente observada e a concentração foi o aspecto do protocolo colocado logo de início em discussão. A acidificação no processo de titulação traz perturbações nas condições analíticas com aumento na força iônica, o que interfere diretamente sobre o comportamento eletroquímico dos metais na análise voltamétrica.

$\mathrm{Na}$ determinação da concentração de cobre ligado ao sulfeto através de VRAPD durante a titulação ácida foram considerados, inicialmente, os valores de corrente obtidos em pH 5,0 e depois da acidificação em $\mathrm{pH}$ 2,8. Entretanto, deve-se ponderar que a acidificação do meio, com mudança de $\mathrm{pH}$ de 5,0 para 2,8, pode representar apenas um melhor sinal analítico para o cobre, ou seja, um aumento na corrente que não significa exclusivamente maior concentração de cobre no meio.

Nesse caso, o ácido nítrico empregado na titulação ácida da amostra aumentou a força iônica do meio, agindo também como eletrólito de suporte e provocando mudanças no comportamento eletroquímico do cobre. $\mathrm{O}$ ambiente iônico potencializado pela presença do eletrólito de suporte aumenta a condutividade da solução, principalmente, em razão das cargas transportadas pelos íons desse composto. ${ }^{12}$

Neste sentido, estudos sistemáticos sobre o comportamento eletroquímico do cobre, durante a titulação ácida, buscaram elucidar 
estas questões e obter dados que permitissem ampliar os conhecimentos sobre a dinâmica do cobre em águas naturais. Novos experimentos foram realizados, em condições simuladas, para verificar a influência do $\mathrm{pH}$ no comportamento eletroquímico do cobre, empregando VRA$\mathrm{PD}$ e os parâmetros já utilizados para as amostras naturais.

Para isso, foram preparadas soluções padrão de cobre (Titrisol Merck) com concentrações de 1,0; 2,5 e 5,0 $\mu \mathrm{g} \mathrm{L}^{-1}$. Alíquotas dessas soluções foram empregadas, em triplicata para cada concentração, determinando-se a corrente em pH 5,0 e 2,8 após acidificação com $\mathrm{HNO}_{3} 0,01 \mathrm{~mol} \mathrm{~L}^{-1}$.

No experimento descrito, empregou-se $\mathrm{KNO}_{3} 0,1 \mathrm{~mol} \mathrm{~L}^{-1}$ como eletrólito de suporte, entretanto pode-se considerar que a adição do ácido na titulação potencializou a ação já exercida pela presença do sal.

Como não há ligantes no meio, qualquer aumento no pico após a acidificação representa influência do aumento da concentração de íons $\mathrm{H}^{+}$no sistema. Verificou-se que os valores de corrente obtidos são maiores em pH 2,8 do que em pH 5,0 para as soluções em todas as concentrações (Tabela 2), encontrando-se uma relação onde após a acidificação, a corrente é aproximadamente o dobro da inicial. Assim sendo, o ácido nítrico adicionado ao sistema assegurou uma concentração hidrogeniônica livre capaz de aumentar a condutividade da solução.

Tabela 2. Variação da corrente em função do $\mathrm{pH}$ para soluções de cobre, concentrações de $1 / 2,5 / 5 \mu \mathrm{g} \mathrm{L}-1$. Ajuste de força iônica com $\mathrm{KNO}_{3} 0,1 \mathrm{~mol}$ $\mathrm{L}^{-1} ; \mathrm{E}_{\text {dep }}=-0,6 \mathrm{~V}(\mathrm{vs} \mathrm{Ag} / \mathrm{AgCl}) ; \mathrm{t}_{\text {dep }}=600 \mathrm{~s}$, empregando-se VRAPD

\begin{tabular}{lcc}
\hline $\mathrm{Cu}\left(\mu \mathrm{g} \mathrm{L}^{-1}\right)$ & $\mathrm{i}(\mathrm{nA})^{*}$ \\
\hline 1,0 & $\mathrm{pH} \mathrm{5}$ & $\mathrm{pH} \mathrm{2,8}$ \\
2,5 & $5( \pm 1)$ & $11( \pm 2)$ \\
5,0 & $19( \pm 2)$ & $45( \pm 2)$ \\
\hline
\end{tabular}

*valores médios das triplicatas; valores entre parênteses indicam estimativa de desvio padrão.

Tendo em vista a influência exercida pelo $\mathrm{pH}$ na corrente observada, torna-se impraticável o cálculo da concentração de cobre ligado ao sulfeto baseado na diferença entre as correntes em pH 5,0 e 2,8.

Considerando estes aspectos, uma nova estratégia foi empregada para se obter um valor mais representativo das reais concentrações de cobre que se dissociam do sulfeto durante o procedimento de titulação ácida.

Para viabilizar esse procedimento, alíquotas de amostra natural filtrada não digerida foram acidificadas para $\mathrm{pH}$ 5,0 e empregando-se VRAPD determinou-se a concentração de cobre na amostra através de titulação, com os seguintes parâmetros: $\mathrm{E}_{\mathrm{i}}=-0,6 \mathrm{~V} ; \mathrm{E}_{\mathrm{f}}=0,15 \mathrm{~V}$; $\mathrm{E}_{\text {cond }}=-1,2 \mathrm{~V} ; \mathrm{t}_{\text {dep }}=600 \mathrm{~s}$.

Como nesse $\mathrm{pH}$ existe a possibilidade de complexação por ligantes naturais presentes na amostra, o procedimento realizado foi o de titulação com solução padrão de cobre $\left(\mathrm{Cu}^{2+}\right)$. Procedeu-se às adições de $\mathrm{Cu}^{2+}$, com auxílio de uma micropipeta diretamente na célula polarográfica, sempre seguidas de períodos de equilíbrio de 20 min para cada adição durante a titulação, e posterior análise. Deve-se salientar ainda que a determinação da concentração de cobre na amostra, antes do início de cada titulação, também foi realizada.

$\mathrm{O} \mathrm{Cu}^{2+}$ inicialmente adicionado é complexado pelos ligantes naturais contidos na amostra, até que se atinja a capacidade máxima de complexação. A partir de então, todo cobre adicionado permanece lábil, é detectado e responsável pela corrente gerada nas análises voltamétricas realizadas.

Dessa maneira, foi possível determinar a concentração de cobre presente na amostra, em $\mathrm{pH}$ 5,0, ou seja, antes da dissociação do cobre ligado ao sulfeto, que ocorre em valores de $\mathrm{pH}$ inferiores a este. Em seguida, outra alíquota foi acidificada para $\mathrm{pH} 2,8$, e a concentração de cobre determinada da mesma forma como relatado para pH 5,0.
Portanto, determinando separadamente o cobre presente na amostra nos dois valores de $\mathrm{pH}$ pode-se obter, pela diferença entre as concentrações, a concentração de cobre que é liberado pela dissociação após acidificação em pH 2,8.

Comparando-se as concentrações de cobre em pH 5,0 e 2,8 e não mais as correntes geradas, o possível erro atribuído ao aumento do sinal analítico do cobre pela acidificação da amostra foi eliminado. Observou-se que a concentração do cobre nas amostras analisadas foi maior em pH 2,8 do que em pH 5,0 evidenciando uma dissociação deste metal em $\mathrm{pH}$ mais ácido.

No entanto, ainda havia outro aspecto a ser observado, o aumento da concentração de cobre após a acidificação para $\mathrm{pH} 2,8$ poderia não estar relacionado exclusivamente à dissociação dos complexos $\mathrm{CuS}$. Como inicialmente abordado, ligantes como a matéria orgânica são importantes agentes complexantes para o cobre e, também, podem estar se dissociando do cobre neste $\mathrm{pH}$.

Segundo Cabaniss e Schuman, ${ }^{13}$ os principais compostos responsáveis pela complexação de metais em ambientes aquáticos são as substâncias húmicas aquáticas, que se encontram na matéria orgânica dissolvida, e como discutido anteriormente, o $\mathrm{pH}$ pode influenciar significativamente no comportamento do conteúdo orgânico dos corpos d'água com relação aos metais investigados. Dessa maneira, novos testes foram realizados para determinar a influência do $\mathrm{pH}$ no comportamento dos complexos Cu-MOD.

\section{Comportamento dos complexos $\mathrm{Cu}-\mathrm{MOD}$ frente à variação de $\mathbf{p H}$}

Pela acidificação, outros complexos formados com o cobre podem dissociar-se da mesma forma que os complexos CuS. Isso pode ocorrer porque com o aumento da concentração de íons $\mathrm{H}^{+}$no meio aquoso, o equilíbrio é deslocado no sentido das formas lábeis do metal. ${ }^{14} \mathrm{Ou}$ seja, em valores mais baixos de $\mathrm{pH}$, a especiação do cobre é afetada pela competição que ocorre entre os íons $\mathrm{H}^{+}$e o cobre por sítios de complexação, sobretudo quando o metal se encontra em baixas concentrações. Assim, a concentração determinada após a acidificação em $\mathrm{pH}$ 2,8 pode incluir também o cobre dissociado dos complexos com a matéria orgânica.

Outro importante aspecto colocado em discussão e estudado neste trabalho com relação ao protocolo inicial foi a questão da complexidade das amostras de águas naturais. Tratando-se de uma matriz complexa, é preciso considerar a presença, na fração dissolvida da amostra, de outros agentes complexantes do cobre, principalmente a matéria orgânica dissolvida, que também pode sofrer dissociação com a acidificação do meio.

O processo de acidificação da amostra para $\mathrm{pH}$ 2,8 com a finalidade de obter a dissociação do cobre ligado ao sulfeto pode ter também, como consequência, a dissociação do cobre complexado pela matéria orgânica.

Buscando verificar esta dissociação e a sua influência nas concentrações de cobre em $\mathrm{pH}$ 2,8 preparou-se uma solução de ácido húmico

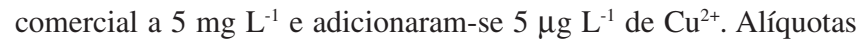
desta solução foram analisadas por VRAPD visando determinar as concentrações de cobre presentes na solução em pH 5,0 e em pH 2,8 após acidificação com $\mathrm{HNO}_{3} 0,1$ mol L $\mathrm{L}^{-1}$, através do método de adição de padrão. Desta forma, pretendeu-se examinar o comportamento dos complexos $\mathrm{Cu}-\mathrm{MOD}$ frente à titulação ácida, observando se ocorre a dissociação destes complexos no mesmo intervalo de $\mathrm{pH}$ que os complexos de sulfeto de cobre, e se esta dissociação contribui para a concentração do metal determinada após acidificação.

Verificou-se após a acidificação que, em média, a concentração de cobre não complexado presente em solução teve um acréscimo de aproximadamente $2 \mu \mathrm{g} \mathrm{L}^{-1}$. Ou seja, a redução do $\mathrm{pH}$ para 2,8 determinou a dissociação de parte do cobre complexado à matéria orgânica. 
Isto ocorre principalmente pela competição entre os íons $\mathrm{H}^{+} \mathrm{e}$ $\mathrm{Cu}^{2+}$ pelos sítios de ligação da matéria orgânica. Em geral, substâncias húmicas tendem a apresentar menor capacidade de complexação com relação ao cátion metálico em valores de $\mathrm{pH}$ baixos, e aumentam a complexação em $\mathrm{pH}$ mais elevados. ${ }^{15}$

Além disso, em águas naturais, apesar da reconhecida capacidade de complexação das substâncias húmicas, outro aspecto que deve ser abordado diz respeito à presença de compostos coloidais inorgânicos na fração dissolvida. A influência da redução do pH nas propriedades químicas dos coloides, bem como nas suas capacidades de complexar ou adsorver metais também devem ser consideradas ao determinar-se a concentração de cobre dissociado pela acidificação da amostra a $\mathrm{pH} 2,8$.

Considerando os resultados obtidos, pode-se afirmar que não é possível estabelecer uma relação direta entre a concentração de cobre dissociado em pH 2,8 e a sua concentração complexada ao sulfeto, pois parte dela pode ser oriunda de complexos orgânicos presentes em amostras naturais.

\section{Construção de curvas analíticas para sulfeto}

A determinação da concentração de cobre ligado ao sulfeto durante o procedimento de titulação ácida só foi possível através da construção de curvas analíticas para o sulfeto. Esta importante adaptação do protocolo proposto por Rozan e colaboradores permitiu a determinação das concentrações de sulfeto em pH 5,0 e 2,8 através de curvas analíticas específicas para cada um destes valores de $\mathrm{pH}$, obtidas a partir de solução padronizada de $\mathrm{Na}_{2} \mathrm{~S} \cdot 9 \mathrm{H}_{2} \mathrm{O}$.

Inicialmente, na etapa de implantação do método para determinação da concentração de sulfeto complexado ao cobre utilizando-se curvas analíticas, empregou-se um tampão TAA (tampão antioxidante alcalino), $\mathrm{pH}$ 13,6 para preparar a solução de $\mathrm{Na}_{2} \mathrm{~S} \cdot 9 \mathrm{H}_{2} \mathrm{O}$ (Vetec A.C.S) buscando-se desta forma uma solução padrão mais estável. Este tampão foi preparado pela dissolução em meio aquoso de $\mathrm{NaOH}$ (Synth - A.C.S), ácido ascórbico (Vetec-A.C.S) e salicilato de sódio (Vetec - A.C.S). Entretanto, nos voltamogramas obtidos foram observados picos interferentes gerados provavelmente por componentes eletroativos do tampão na região de potenciais relacionados ao sulfeto $(-0,6 \mathrm{~V})$, fato que inviabilizou o preparo do padrão empregando solução tampão. Optou-se, então, pelo preparo da solução padrão em meio aquoso, no momento da sua utilização.

Para obtenção da curva analítica empregou-se VRAOQ e os mesmos parâmetros instrumentais utilizados para sulfeto em amostras naturais. A solução de sulfeto, preparada no momento da titulação, foi padronizada com auxílio de um eletrodo tipo redox Analion $\mathrm{Ag}_{2} \mathrm{~S} /$ $\mathrm{S}^{2-}$, modelo 064, conectado a um potenciômetro Micronal B474, e descartada após utilização para uma única amostra.

Foram construídas curvas em pH 5,0 e 2,8 específicas para cada amostra coletada, relacionando a corrente obtida por VRCOQ com a concentração de sulfeto. As alíquotas reservadas para as curvas foram acidificadas e submetidas à purga com $\mathrm{N}_{2}$ antes da adição de sulfeto.

Alíquotas em volume reduzido foram adicionadas diretamente na célula polarográfica. $\mathrm{O}$ experimento foi realizado em batelada empregando-se uma célula para cada concentração. Os parâmetros instrumentais empregados na VRCOQ foram os mesmos citados anteriormente para sulfetos ${ }^{14} \mathrm{e}$ as curvas analíticas obtidas estão exemplificadas na Figura 2.

As concentrações de sulfeto empregadas na construção das curvas estiveram entre 1,0 e $25,0 \mu \mathrm{g} \mathrm{L} \mathrm{L}^{-1}$ para o volume de $10 \mathrm{~mL}$ da amostra contido na célula polarográfica, variando conforme a corrente inicial observada para o sulfeto em cada amostra. Estas concentrações foram obtidas diretamente na célula polarográfica pela adição de solução padrão, preparada e padronizada no momento da análise, às alíquotas

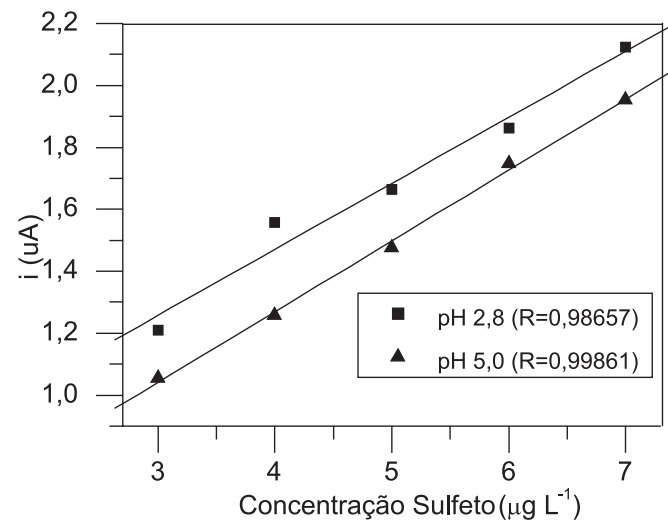

Figura 2. Curvas analíticas para determinação de sulfeto, obtidas por VRCOQ durante titulação ácida

de amostra natural, filtradas e acidificadas para os valores específicos de $\mathrm{pH}$ e submetidas à purga com $\mathrm{N}_{2}$. A concentração da solução de sulfeto de sódio foi distinta para cada amostra, de forma que o volume máximo adicionado à célula fosse $500 \mu \mathrm{L}$.

Conhecendo-se as correntes obtidas para a amostra em pH 5,0 e 2,8 aplica-se a equação da reta em cada uma das curvas analíticas, e obtêm-se as concentrações de sulfeto em pH 5,0 e 2,8. Pela diferença calcula-se a concentração de sulfeto ligado ao cobre e a razão estequiométrica entre cobre e sulfeto permite determinar a concentração de cobre dissociado.

As características dos métodos voltamétricos, principalmente quanto à sensibilidade, associadas à construção de curvas analíticas, recomendam seu emprego no estudo da dinâmica e do comportamento do cobre frente a sulfetos solúveis em ambientes aquáticos. A aplicação do protocolo de titulação ácida, a partir das modificações implantadas neste trabalho, viabilizou a detecção de níveis reduzidos não apenas do metal como também de sulfeto. A aplicação do método a amostras de água provenientes de quatro rios localizados na Região Metropolitana de Curitiba permitiu a determinação das concentrações de sulfeto dissociado do cobre, com resultados que variaram de 2 a $131 \mathrm{nmol} \mathrm{L}^{-1}$, evidenciando diferenças em função dos níveis de ocupação da bacia.

\section{CONCLUSÕES}

O protocolo implantado neste trabalho a partir das modificações necessárias permitiu a avaliação da complexação de cobre por sulfetos em águas naturais, através de titulação ácida e do uso de métodos voltamétricos e mostrou-se adequado para os objetivos pretendidos. $\mathrm{O}$ sulfeto de cobre foi identificado e quantificado em águas superficiais empregando-se VRCOQ, com eletrodo de mercúrio de gota pendente, na faixa de nmol L-1, através de curvas analíticas.

Os resultados ratificam o que tem sido observado nas determinações de complexos $\mathrm{CuS}$ e estão de acordo com os recentes relatos na literatura, confirmando que é preciso considerar a importância da participação dos sulfetos solúveis como agentes complexantes para a compreensão dos aspectos que governam a especiação de metais em águas naturais.

\section{REFERÊNCIAS}

1. Scheffer, E. W. O.; Sodré, F. F.; Grassi, M. T.; Quim. Nova 2007, 30, 332.

2. Sukola, K.; Wang, F.; Tessier, A.; Anal. Chim. Acta 2005, 528, 183.

3. Bowles, K. C.; Ernste, M. J.; Kramer, J. R.; Anal. Chim. Acta 2003, 477, 113. 
4. Rozan, T. F.; Lassman, M. E.; Ridge, D. P.; Luther, G. W.; Nature 2000, 406, 879.

5. Rozan, T. F.; Benoit, G.; Geochim. Cosmochim. Acta 1999, 63, 3311.

6. Luther III, G. W.; Tsamakis, E.; Mar. Chem. 1989, 27, 165.

7. Locke, D. C.; He, Y.; Zheng, Y.; Anal. Chim. Acta 2002, 459, 209.

8. Mylon, S. E.; Twining, B. S.; Fisher, N. S.; Benoit, G.; Environ. Sci. Technol. 2003, 37, 1261.

9. Ciglenecki, I.; Kodba, Z.; Cosovic, B.; Mar. Chem. 1996, 53, 101.

10. Rozan, T. F.; Benoit, G.; Luther III, G. W.; Environ. Sci. Technol. 1999, 33,3021 .
11. Namiesnik, J.; Sliwka-Kaszynska, M.; Kot-Wasik, A.; Environ. Sci. Technol. 2003, 33, 31.

12. Skoog, D. A.; West, D. M.; Holler, F. J.; Crouch, S. R.; Fundamentos de Química Analítica, $8^{\text {a }}$ ed., Grassi, M. T., trad.; Pioneira Thomson Learning: São Paulo, 2006

13. Cabaniss, S. E.; Shuman, M. S.; Geochim. Cosmochim. Acta 1988, 52, 185.

14. Gundersen, P.; Steinnes, E.; Water Res. 2003, 37, 307.

15. Zuyi, T.; Taiwei, C.; Jinzhou, D.; XiongXing, D.; Yingiie, G.; Appl. Geochem. 2000, 15, 145. 